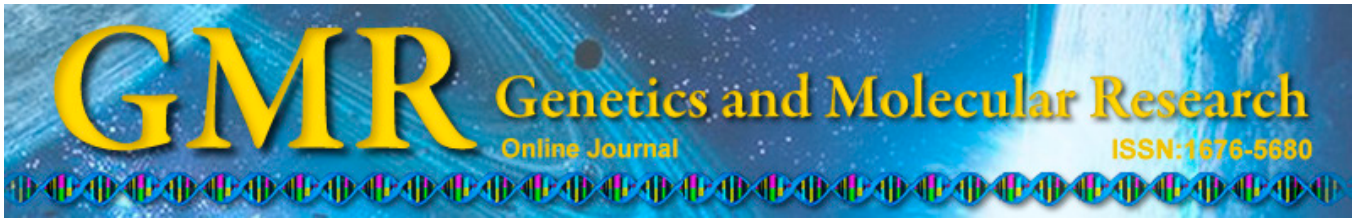

\title{
Cloning and characterization of peanut allene oxide cyclase gene involved in salt-stressed responses
}

\author{
H.H. Liu ${ }^{1 *}$, Y.G. Wang ${ }^{2 *}$, S.P. Wang ${ }^{3}$ and H.J. Li $^{3}$ \\ ${ }^{1}$ Postdoctoral Research Center of Shandong Shengfeng Seeds Co., Ltd., \\ Jiaxiang, Shandong, China \\ ${ }^{2}$ Institute of Genetics and Developmental Biology, Chinese Academy of Sciences, \\ Beijing, China \\ ${ }^{3}$ Shandong Shengfeng Seeds Co., Ltd., Jiaxiang, Shandong, China \\ *These authors contributed equally to this study. \\ Corresponding author: Y.G. Wang \\ E-mail: ygwang@genetics.ac.cn
}

Genet. Mol. Res. 14 (1): 2331-2340 (2015)

Received May 6, 2014

Accepted October 23, 2014

Published March 27, 2015

DOI http://dx.doi.org/10.4238/2015.March.27.18

\begin{abstract}
In this study, the full-length cDNA encoding allene oxide cyclase $(A h A O C)$ was isolated from peanut (Arachis hypogaea L.). The deduced amino acid sequence of $A h A O C$ showed high homology with other plant AOCs. The transcript of $A h A O C$ was found to be abundantly expressed in roots. Expression analysis demonstrated that $A h A O C$ was induced by abscisic acid, methyl-jasmonic acid, salicylic acid, salinity, polyethylene glycol, and cold stresses, particularly by high salinity. Overexpression of $A h A O C$ in rice increased root elongation and plant height compared with expression in control plants and conferred tolerance against salinity. Thus, the $A h A O C$ gene may play an important role in increasing the expression of transcription factors (MYB2 and OsONAC045) and functional genes (DREB1F and LEA3) in transgenic rice under salt stress as well as improve stress tolerance through the
\end{abstract}


accumulation of compatible solutes (proline and soluble sugar). The $A h A O C$ gene is a potential resource for enhancing salt tolerance in crop species.

Key words: Allene oxide cyclase gene; Gene cloning; Overexpression; Peanut; Salt stress

\section{INTRODUCTION}

During plant adaptation to environmental stresses, such as pathogens, salinity, drought, and high light levels, various signals are produced, including jasmonic acid (JA). JA is an important endogenous plant hormone involved in various stress responses (Creelman and Mullet, 1997; Parchmann et al., 1997). In plants, JA biosynthesis occurs through the oxylipin pathway in the chloroplasts (Stumpe and Feussner, 2006). Molecular oxygen is added to linolenic acidin in a reaction catalyzed by lipoxygenase, forming (13S)-hydroperoxy$(9 Z, 11 E, 15 Z)$-octadecatrienoic acid. This product is then dehydrated by allene oxide synthase (AOS, EC 4.2.1.92) to an unstable allene oxide; this reaction is catalyzed by allene oxide cyclase (AOC, EC 5.3.99.6) to the stable compound 12-oxo-phytodienoic acid. After a 12-oxophytodienoic acid reductase reaction and 3 cycles of $\beta$-oxidation, (+)-7-iso-JA is produced. $\mathrm{AOC}$ is an important enzyme in JA biosynthesis as it determines the correct stereochemical structure of JA (Ziegler et al., 2000). Therefore, $A O C$ genes are thought to be potential targets for controlling metabolites biosynthesis in plant species.

There are $24 A O C$ gene sequences deposited in the NCBI GenBank database, of which 6 are from soybean, 4 from Arabidopsis thaliana, and only 2 from common wheat and maize genomes. Characterization of $A O C$ genes from various plant species has provided insight into their biochemical and physiological roles in adaptation to biotic and abiotic stresses. For example, overexpression of $J c A O C$ in Escherichia coli improved its resistance to salt and low temperature stresses (Liu et al., 2010). Wu et al. (2011) examined the expression and functional diversity of the $A O C$ gene family in soybean. They found that $6 A O C$ genes had specific and different expression patterns in multiple organs and under several stresses. Overexpression of the $\mathrm{GmAOCl}$ and $\mathrm{GmAOC} 5$ genes in transgenic tobacco improved tolerance to salt and oxidative stresses, respectively. However, the precise mechanisms of $A O C$ function in plant cell processes, particularly in regulating stress response, are not well-understood.

Plant $A O C$ had specific and complex expression patterns in multiple organs. In soybean, GmAOC2 shows relatively higher expression only in roots, GmAOC5 is only strongly expressed in stems, and GmAOC3 is expressed highly in flowers (Wu et al., 2011). In tomato, the $A O C$ gene is specifically expressed in vascular bundles and surrounding parenchymatic cells (Hause et al., 2000). In Leymus mollis, LmAOC expression is highly induced by drought, heat, cold, and wounding, as well as by JA and abscisic acid (ABA) (Eltayeb Habora et al., 2013). Four $A O C$ genes in A. thaliana are constitutively expressed in all leaf tissues (Stenzel et al., 2003). Although some plant $A O C$ genes have been characterized in many plant species, the role of peanut $A O C$, particularly in stress tolerance, remains unknown.

Cultivated peanut (Arachis hypogaea L.) is one of the most important oilseed crops worldwide. It is widely cultivated in tropical, subtropical, and temperate climates. Like other crops, peanut is susceptible to many adverse environmental stresses. Therefore, understanding the molecular basis of stress responses is a key target for peanut genetic improvement 
programs. In the present study, we isolated a novel $A O C$ gene from peanut and transferred the gene into rice to increase salinity resistance in transgenic rice by overexpression of the protein.

\section{MATERIAL AND METHODS}

\section{Plant materials, growth conditions, and treatments}

Peanut cultivars (A. hypogaea L., 'Luhua 14') seeds were germinated at $23^{\circ} \mathrm{C}$ for 1 week, and then the germinated seedlings were transferred to plastic flats containing $15 \mathrm{~L}$ nutrient solution. The nutrient solution contained (in $\mathrm{mM}$ ) $0.2 \mathrm{KH}_{2} \mathrm{PO}_{4} ; 0.5 \mathrm{MgSO}_{4} ; 1.5 \mathrm{KCl} ; 1.5$ $\mathrm{CaCl}_{2} ; 1 \times 10^{-3} \mathrm{H}_{3} \mathrm{BO}_{3} ; 5 \times 10^{-5}\left(\mathrm{NH}_{4}\right)_{6} \mathrm{Mo}_{7} \mathrm{O}_{24} ; 5 \times 10^{-4} \mathrm{CuSO} 4 ; 1 \times 10^{-3} \mathrm{ZnSO}_{4} ; 1 \times 10^{-3} \mathrm{MnSO}_{4}$; $1 \times 10^{-1} \mathrm{Fe}(\mathrm{III})-\mathrm{EDTA}$. The seedlings were grown in a controlled environment growth chamber under the following conditions: $16 \mathrm{~h}$ light $/ 8 \mathrm{~h}$ dark photoperiod with a light intensity of 400 $\mu \mathrm{M} \mathrm{m}^{-2} \mathrm{~s}^{-1}, 23^{\circ} \mathrm{C}$ day and night temperatures, and relative humidity of $60-75 \%$. Two-week-old seedlings with similar heights were transferred to nutrient solution containing $100 \mu \mathrm{M}$ ABA, $50 \mu \mathrm{M}$ methyl-jasmonic acid (MeJA), $50 \mu \mathrm{M}$ salicylic acid (SA), $120 \mathrm{mM} \mathrm{NaCl}$, and $20 \%$ polyethylene glycol (PEG) 6000, respectively. For cold stress treatments, plants were placed in a dark chamber at $4^{\circ} \mathrm{C}$. At $0,3,8,12,24,36$, and $72 \mathrm{~h}$ after beginning treatment, the leaves were quickly frozen in liquid nitrogen and stored at $-70^{\circ} \mathrm{C}$ until use.

Field grown plants of Luhua14 were used to analyze tissue-specific expressions of $A h A O C$. At 15 days after flowering, leaf, stem, root, flower, and gynophore tissues were sampled for gene expression analysis.

\section{Isolation, sequencing, and analysis of $A h A O C$}

AhAOC gene was obtained using an in silico cloning strategy. The reported nucleotide sequence of $A O C$ (NCBI: EU146298) from rice was used as a query sequence to search the peanut expressed sequence tag database (http://www.ncbi.nlm. nih.gov) using the blastn program. Based on the resulting sequence of interest, primer pairs (forward primer: 5'-CACACACCCCAATAACCTTCC-3', and reverse primer: 5'-AGTCAGTGAAACCAGAAATAAC-3') were designed to amplify the cDNA sequences of $A h A O C$ in Luhua 14. Polymerase chain reaction (PCR) was performed using Pfu DNA polymerase (Promega, Madison, WI, USA). The gene isolated was sequenced by ABI3730 DNA Analyzer (Applied Biosystems, Inc., Foster City, CA, USA).

\section{Gene structure and bioinformatic analysis}

The DNAMAN (5.2.2) software was used for sequence analysis. The coding region was identified using the open reading frame finder (http://www.ncbi.nlm.nih.gov/gorf/gorf. html). AhAOC homologs were identified using the NCBI BLAST server.

\section{Real-time quantitative PCR and semi-quantitative reverse transcription (RT)- PCR analysis}

Total RNA was extracted from peanut and rice using the AxyPrep ${ }^{\mathrm{TM}}$ Multisource Total RNA Miniprep Kit (Axygen, Union City, CA, USA) and treated with RNase-free DNase (Ta- 
kara, Shiga, Japan). First-strand cDNA was synthesized using oligo-(dT) ${ }_{18}$ as an anchor primer and M-MLV reverse transcriptase (Promega) according to manufacturer instructions. AhActin and OsActin were used as internal reference genes to assess expression levels in peanut and rice, respectively, and the primers for these genes were specific for amplifying a single gene. Real-time quantitative PCR was conducted by using the SYBR ${ }^{\circledR}$ Premix Ex Taq ${ }^{\mathrm{TM}}$ II (Takara) in a Mastercycler ${ }^{\circledR}$ ep realplex (Eppendorf, Hamburg, Germany). Each $20-\mu \mathrm{L}$ sample was subjected to 40 cycles at $94^{\circ} \mathrm{C}$ for $5 \mathrm{~s}$ and $60^{\circ} \mathrm{C}$ for $20 \mathrm{~s}$. Triplicate quantitative assays were performed for each cDNA sample. The relative expression of $A h A O C$ was detected using the $2^{-\Delta \Delta \mathrm{CT}}$ method (Livak and Schmittgen, 2001). For semi-quantitative RT-PCR, the following program was used: 5 min denaturation at $94^{\circ} \mathrm{C}$, followed by 30 cycles of $94^{\circ} \mathrm{C}$ for $30 \mathrm{~s}, 55^{\circ} \mathrm{C}$ for $30 \mathrm{~s}$, and $72^{\circ} \mathrm{C}$ for $30 \mathrm{~s}$, with a final extension step for $8 \mathrm{~min}$ at $72^{\circ} \mathrm{C}$. The primer sequences used for semi-quantitative RT-PCR and real-time quantitative PCR are listed in Table1.

Table 1. Primer sequences used for real-time quantitative PCR and semi-quantitative RT-PCR.

\begin{tabular}{lllll}
\hline Name & Sequence (5' to 3') & Name & Sequence (5' to 3') & Function \\
\hline AhActin- $F$ & GTGGCCGTACAACTGGTATTGT & AhActin- $R$ & TGGATGGCTGGAAGAGAACT & real-time quantitative PCR \\
AhAOC-F & GTATGAGATCAATGAGAGAGAT & AhAOC-R & TACCTGTCCCCTTTCTTCTCTG & semi-quantitative RT-PCR \\
OsACTIN $-F$ & CCTCGTCTCGACCTTGCTGGG & OsACTIN1-R & GAGAACAAGCAGGAGGACG & real-time quantitative PCR \\
OsDREB $1 F-F$ & GGCGGAAGAAATTCCGGGAG & OsDREB $1 F-R$ & GCGATGGCCGCGACGTCGTG & real-time quantitative PCR \\
Os $L A 3-F$ & AGACCAAGGCCCACACCGAG & OsLEA3- $R$ & CCGTCTCCGACGCCCTCTC & real-time quantitative PCR \\
OsONAC045-F & ATATGCCTCCACCTCCTCCT & OsONAC045-R & CATCTCGTAGGGCTTTCACC & real-time quantitative PCR \\
Os $M Y B 2-F$ & GGCTGAAACGCACAGGCAAG & OsMYB2- $R$ & CTGCTTG GCGTGCTTCTGC & real-time quantitative PCR \\
\hline
\end{tabular}

\section{Vector construction and plant transformation}

The full-length $A h A O C$ coding sequence was amplified using the primers 5'-GGGGTACCATGGCTTCCTCATCAACTACTTTTG-3' (forward, KpnI site underlined) and 5'-GCGAGCTCTCAGTGAAACCAGAAATAACAGCATG-3' (reverse, SacI site underlined). The coding region was cloned into the $K p n I$ and $S a c I$ sites of the pUN1301 vector under control of the ubiquitin promoter. The pUN1301-AhAOC construct was transferred into A. tumefaciens EHA105 cells and then introduced into rice embryonic calli and $\mathrm{T}_{1}$ transgenic plants were confirmed by PCR analysis using gene-specific primers of $A h A O C$ as described above.

\section{Physiological analysis of plants expressing $A h A O C$ under salt stress}

For salt treatment, seeds from wild-type and $2 \mathrm{~T}_{3}$ generation $A$ AOOC-overexpressing rice lines (OE11 and OE13) were grown on MS medium containing 0,80 , and $120 \mathrm{mM} \mathrm{NaCl}$. After 1 week, root length of 20 plants was measured. For seedling treatment with salt stress, 3 -week-old plants grown in pots were treated with $120 \mathrm{mM} \mathrm{NaCl}$ for 2 weeks. Next, the leaves of wild-type and transgenic lines were collected. JA, proline, and soluble sugar content were measured as described previously by Dong et al. (2013), Bailey et al. (1958), and Shan et al. (2007), respectively.

\section{Statistical analysis}

Data was analyzed using analysis of variance. 


\section{RESULTS}

\section{$A h A O C$ gene cloning and sequence analysis}

A 852-bp cDNA fragment was amplified by RT-PCR and referred to as AhAOC. Present in the cDNA fragment was a 105-bp 5'-untranslated region, 746-bp coding region, and 1-bp 3'-untranslated region. The deduced peptide consisted of 248 amino acids with a molecular mass of 27,263.0 Da and $\mathrm{pI}$ of 9.46. Multi-alignment analysis revealed that the protein shared 70, 66, 82, and 67\% amino acid identity with those in soybean (HM803106.1), Hordeum vulgare (CAC83766.1), Oryza sativa (CAD38519), wheat (AGU41846), and Zea mays (AAR33049) (Figure 1).

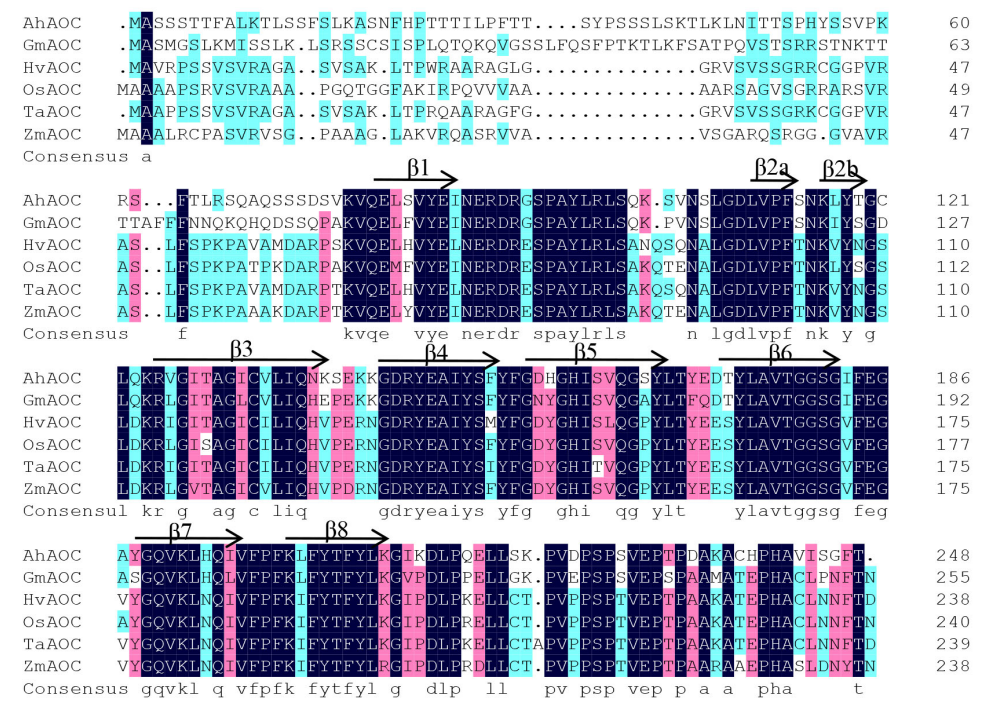

Figure 1. An alignment is shown for the deduced amino acid sequence of area under the curve from peanut, soybean, barley, rice, wheat, and maize. Residues that were identical in all proteins are shown with a black background. The arrows indicate $8 \beta$-strands. The numbers on the right indicate the amino acid position. Protein accession numbers are as follows: ZmAOC, AAR33049; HvAOC, CAC83766.1; OsAOC, CAD38519; GmAOC, HM803106.1; TaAOC, AGU41846.

\section{Expression pattern of $A h A O C$ in different tissues and response to abiotic stresses}

We first analyzed tissue-specific expression of $A h A O C$ in Luhua 14 at 15 days after flowering. Transcripts of $A h A O C$ under field conditions were primarily observed in the root, leaf, and root, with less expression in the stem and gynophores (Figure 2). We next examined the transcriptional responses of $A h A O C$ under multiple abiotic stresses, such as MeJA, ABA, $\mathrm{SA}$, cold $\left(4^{\circ} \mathrm{C}\right), 120 \mathrm{mM} \mathrm{NaCl}$, and 20\% PEG6000 (Figure 3). AhAOC showed different transcript regulation patterns under different hormone conditions (Figure $3 \mathrm{~A}-\mathrm{C}$ ). In the presence of MeJA, the expression of $A h A O C$ increased from 0-36 h, reached a maximum at $12 \mathrm{~h}$, and then decreased sharply at $72 \mathrm{~h}$ (Figure 3A). Treatment with ABA led to a significant increase in transcript levels within $72 \mathrm{~h}$ and was the highest at $36 \mathrm{~h}$ (Figure 3B). SA significantly improved 
the mRNA level within first $12 \mathrm{~h}$, then reduced at $24 \mathrm{~h}$, but quickly and remarkably induced transcripts again at $36 \mathrm{~h}$, followed by a quick reduction at $72 \mathrm{~h}$ (Figure 3C). Cold did not significantly change transcription levels within $12 \mathrm{~h}$, but was followed by significant increased levels, peaking at $24 \mathrm{~h}$ (Figure 3D). During the treatment period, $\mathrm{NaCl}$ stress markedly increased expression levels of the $A h A O C$ gene, peaking at $8 \mathrm{~h}$ (Figure $3 \mathrm{E}$ ). PEG-induced stress did not alter transcript levels during the first $3 \mathrm{~h}$ under treatment, but greatly increased transcription levels at $12 \mathrm{~h}$, followed by a quick reduction to its basal levels at $72 \mathrm{~h}$ (Figure $3 \mathrm{~F}$ ).

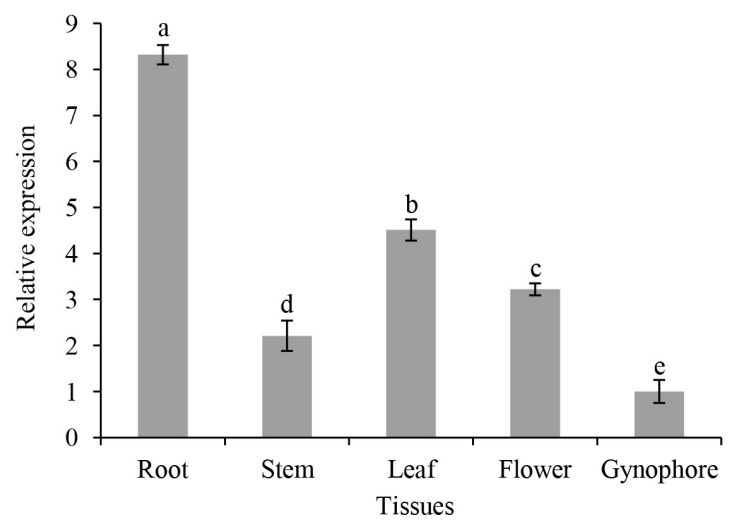

Figure 2. Relative expression levels of $A h A O C$ in different tissues. Data are reported as means $\pm \mathrm{SE}$ of 3 biological replicates. Each replicate contained 10 plants. Different letters above the column indicate statistical significance at the 0.05 probability level.
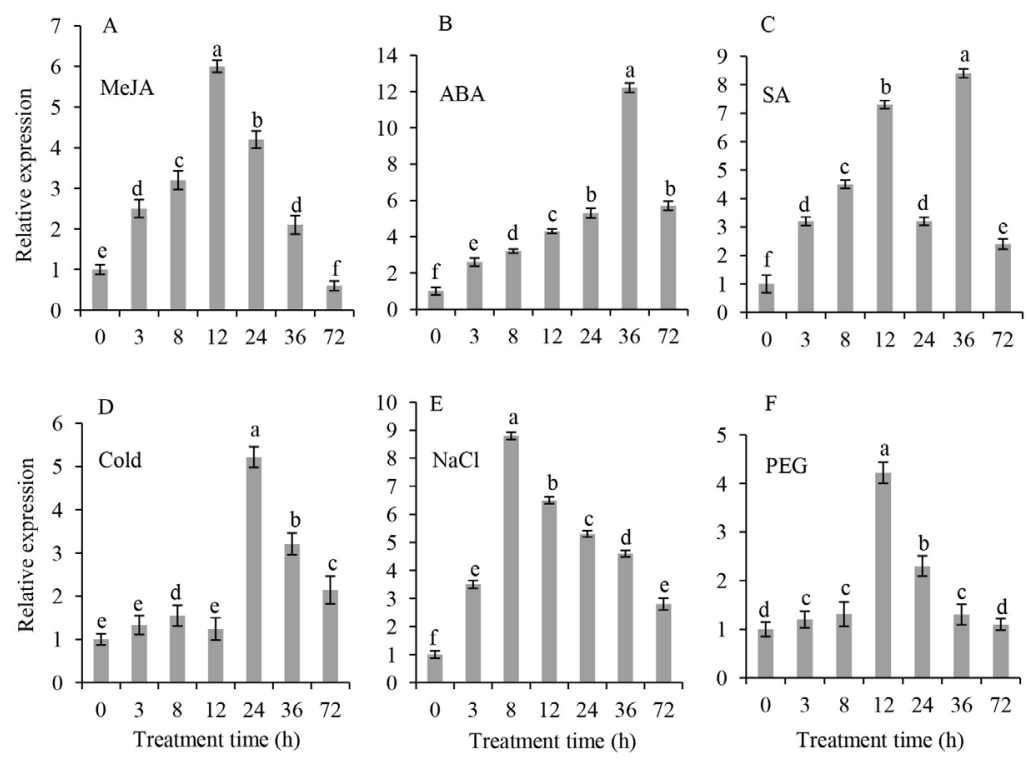

Figure 3. Expression profiles of TaAOC response to MeJA, ABA, SA, cold, NaCl, and 20\% PEG6000. Each replicate contained ten plants. Different letters above the column indicate statistical significance at the 0.05 probability level. 


\section{$A h A O C$ overexpression in rice results in enhanced tolerance to salt stress}

The mRNA level of AhAOC was strongly increased in the $2 \mathrm{~T}_{3}$ transgenic lines OE11 and OE13 (Figure 4A). To determine the salt tolerance of transgenic rice, $A$ AOOC-overexpressing and wild-type seeds were germinated on MS plates supplemented with 0,80 , or 120 $\mathrm{mM} \mathrm{NaCl}$. The high concentrations of $\mathrm{NaCl}$ inhibited root growth, but the transgenic lines showed longer root lengths (Figure 4B and C). We next examined the salt tolerance of rice seedlings from the 2 overexpression lines. The seedlings were cultured in pots for 3 weeks and were subsequently treated with $120 \mathrm{mM} \mathrm{NaCl}$. After salt treatment for 2 weeks, most of the wild-type seedlings were wilting and yellow. Under control conditions $(0 \mathrm{mM} \mathrm{NaCl})$, there was no significant difference in plant heights between the wild-type and transgenic plants. After the plants were treated with $\mathrm{NaCl}$ for 2 weeks, plant heights reduced significantly. Moreover, this reduction was greater in wild-type than in the transgenic plants (Figure 4E). In addition, the contents of JA, soluble sugar, and proline were higher in AhAOC-overexpressing plants under salt stress (Figure 4F-H).
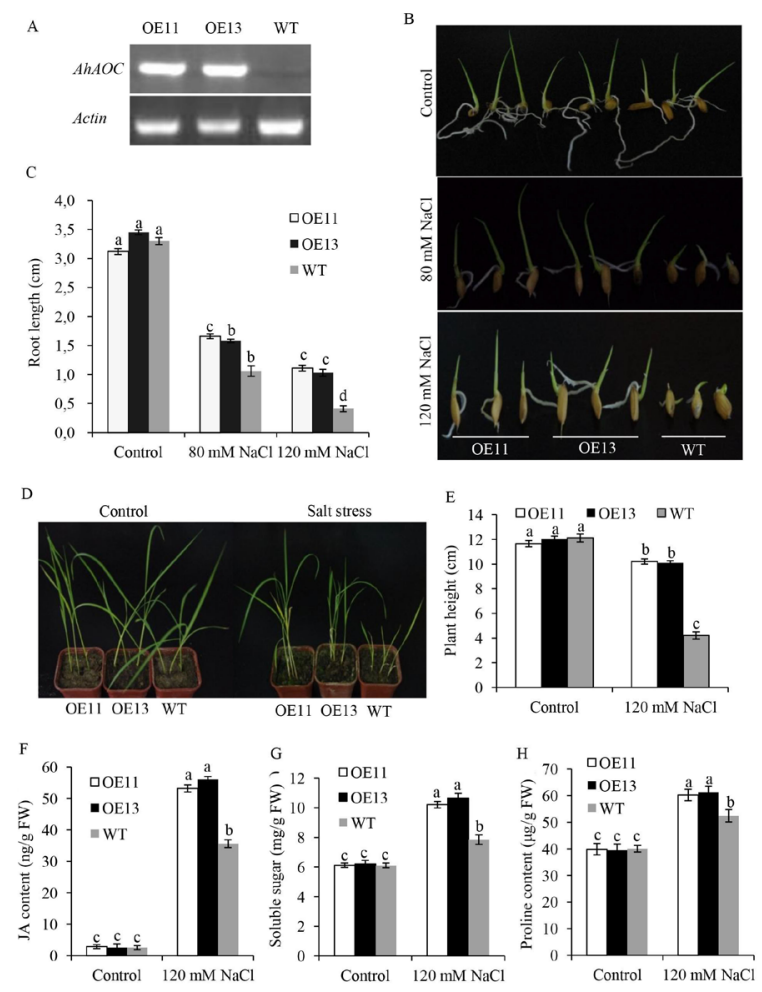

Figure 4. Analysis of salt tolerance in $A h A O C$-overexpressing rice. A. Semi-quantitative RT-PCR analysis of the $A h A O C$ expression in wild-type (WT) and transgenic plants. B. Germination phenotypes of WT and transgenic plants on medium containing 0,80 , or $120 \mathrm{mM} \mathrm{NaCl}$ for 1 week. C. Root lengths of WT and $A$ hAOC-overexpressing plants on medium containing 0,80 , or $120 \mathrm{mM} \mathrm{NaCl}$ for 1 week. D. Seedling growth performance of WT and AhAOC-overexpressing plants after 3 weeks with $120 \mathrm{mM} \mathrm{NaCl}$. E. Plant heights, JA, soluble sugar, and proline content in transgenic and WT plants exposed to $120 \mathrm{mM} \mathrm{NaCl}$ for 3 weeks. Different letters above the column indicate statistical significance at the 0.05 probability level. 


\section{Expression of stress-associated genes in transgenic rice}

The relative expression levels of several known stress-associated marker genes $O s$ DREB1F, OSLEA3, OSMYB2, and OSONAC045(Moons et al., 1995; Wang et al., 2008; Zheng et al., 2009; Yang et al., 2012) were examined in $2 \mathrm{~T}_{3}$ generation $A h A O C$-overexpressing rice lines using real-time quantitative PCR. All genes exhibited increased expression levels in 2 transgenic lines with salt treatment compared with control plants (Figure 5).

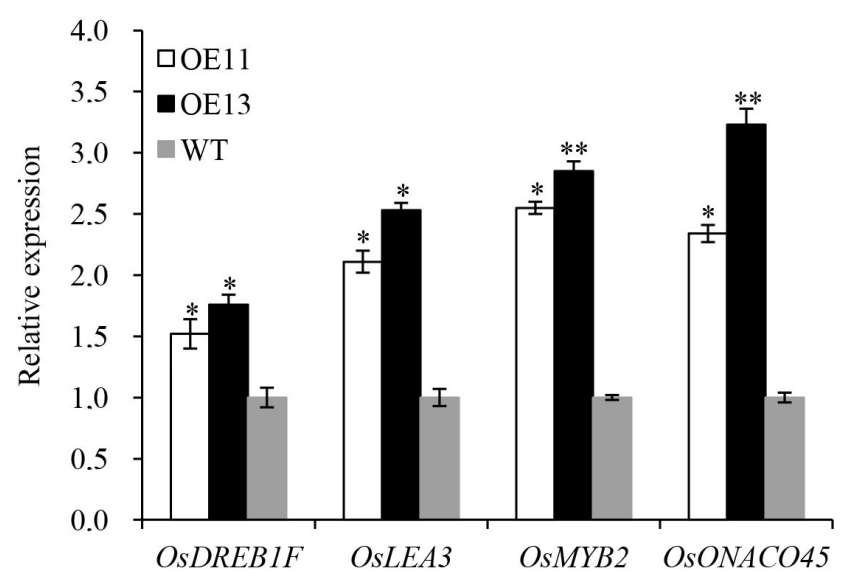

Figure 5. Expression patterns of 4 stress-responsive genes in response to salt treatment. Each column represents an average of 3 replicates. ** and * indicate significant differences compared with WT at $\mathrm{P}<0.01$ and 0.05 , respectively.

\section{DISCUSSION}

In the present study, we cloned the full-length cDNA of $A h A O C$ containing an open reading frame of $746 \mathrm{bp}$ and encoding a protein of 248 amino acids from the cultivated peanut Luhua 14. Analysis of tissue-specific expression revealed AhAOC expression in 5 different tissues, with the highest relative expression in the roots (Figure 2A). A previous study in soybean also found that GmAOC2 was predominantly expressed in the roots (Wu et al., 2011). This is reasonable because roots are buried in soil and can sense the presence of salt. The dynamic expression patterns of $A h A O C$ were affected by cold $\left(4^{\circ} \mathrm{C}\right), \mathrm{NaCl}$, and $20 \%$ PEG6000 (Figure 3), indicating that $A h A O C$ may be involved in the cross-talk of the multi-environmental stress response in peanut. ABA, MeJA, and SA are important cellular regulators in the response to various environmental stresses in plants (Grill and Himmelbach, 1998; Wasternack and Hause, 2002; Horváth et al., 2007). AhAOC was temporally regulated by ABA, SA, and MeJA treatments (Figure 3), suggesting that upregulation of $A h A O C$ in the presence of $\mathrm{NaCl}$, cold, and 20\% PEG6000 may be associated with ABA-, SA-, and MeJA-mediated signaling pathways. Further studies should be conducted to examine the molecular mechanism through which the $A h A O C$ protein interacts with the signal transduction systems of these 3 hormones.

In the current study, we found that overexpression of $A h O C$ in rice significantly improved salt stress tolerance. This tolerance was supported by phenotypic performance and 
physiological indices changes. Root elongation was significantly higher in transgenic lines compared to in wild-type plants (Figure 4B). Plants may enhance stress tolerance by accumulating osmolytes, such as soluble sugars and free proline, to protect cell structures (Szabados and Savouré, 2010; Cui et al., 2011). The increased soluble sugars and proline content under environmental stresses significantly enhanced plant stress tolerance (Abrahám et al., 2003; Verbruggen and Hermans, 2008; Cui et al., 2011; Cvikrová et al., 2012). To investigate the potential mechanisms underlying the improved stress tolerance of $A h A O C$ in overexpressed plants, soluble sugars and free proline levels were measured under salt stress conditions. The results indicated that the content of soluble sugars and free proline in the transgenic plants were significantly higher than those in wild-type plants under salt stress (Figure 5). Therefore, higher tolerance against salt stresses in transgenic rice may result from osmolytes accumulation. To analyze the improved salt tolerance at the molecular level, expression of 4 stress-responsive genes (MYB2, ONAC045, DREB1F, and LEA3) were examined between transgenic plants and controls. Overexpression of $A h A O C$ in rice led to higher expression of all 4 stress-related genes (Figure 6). It has been found that the transcription factors $M Y B 2$ and OsONAC045 were involved in ABA-inducible gene expression, OsDREB $1 F$ and OsLEA3 maybe induced by different stresses through an ABA-dependent pathway, and they also play positive roles in salt tolerance (Moons et al., 1995; Wang et al., 2008; Zheng et al., 2009; Yang et al., 2012). AhAOC may act as an activator to increase the expression levels of transcription factors $(O S M Y B 2$ and OsONACO45) and functional genes (OSDREB1F and OsLEA3) under salt stress condition.

In conclusion, we identified and characterized a novel $A h A O C$ gene from cultivated peanut. $A h A O C$ was differentially expressed in response to diverse stresses and hormones, and overexpression of $A h A O C$ led to enhanced tolerance to salt stress in transgenic rice. $A h A O C$ may be useful for molecular breeding of salt tolerant crops, such as peanut, wheat, maize, and other gramineous plants.

\section{ACKNOWLEDGMENTS}

Research supported by the project of Modern Seed Industry Enterprise Science and Technology Development of Shandong, China (\#SDKJ2012QF003).

\section{REFERENCES}

Abrahám E, Rigó G, Székely G, Nagy R, et al. (2003). Light-dependent induction of proline biosynthesis by abscisic acid and salt stress is inhibited by brassinosteroid in Arabidopsis. Plant Mol. Biol. 51: 363-372.

Bailey RW (1958). The reaction of pentoses with anthrone. Biochem. J. 68: 669-672.

Creelman RA and Mullet JE (1997). Biosynthesis and action of jasmonates in plants. Ann. Rev. Plant Physiol. Plant Mol. Biol. 48: 355-381.

Cui M, Zhang W, Zhang Q, Xu Z, et al. (2011). Induced overexpression of the transcription factor OsDREB2A improves drought tolerance in rice. Plant Physiol. Bioch. 49: 1384-1491.

Cvikrová M, Gemperlová L, Dobrá J, Martincová O, et al. (2012). Effect of heat stress on polyamine metabolism in proline-overproducing tobacco plants. Plant Sci.182: 49-58.

Dong W, Wang MC, Xu F, Quan TY, et al. (2013). Wheat oxophytodienoate reductase gene TaOPRI confers salinity tolerance via enhancement of abscisic acid signaling and reactive oxygen species scavenging. Plant Physiol. 161: $1217-1228$.

Eltayeb Habora MEE, Eltayeb AE, Oka M, Tsujimoto H, et al. (2013). Cloning of allene oxide cyclase gene from Leymus mollis and analysis of its expression in wheat-Leymus chromosome addition lines. Breed. Sci. 63: 68-76. 
Grill E and Himmelbach A (1998). ABA signal transduction. Curr. Opin. Plant Biol.5: 412-418.

Hause B, Stenzel I, Miersch O, Maucher H, et al. (2000). Tissue-specific oxylipin signature of tomato flowers: allene oxide cyclase is highly expressed in distinct flower organs and vascular bundles. Plant J. 24: 113-126.

Horváth E, Szalai G and Janda T (2007). Induction of abiotic stress tolerance by salicylic acid signaling. J. Plant Growth Regul. 26: 290-300.

Liu B, Wang WG, Gao JH, Chen F, et al. (2010). Molecular cloning and characterization of a jasmonate biosynthetic pathway gene for allene oxide cyclase from Jatropha curcas. Acta Physiol. Plant. 32: 531-539.

Livak KJ and Schmittgen TD (2001). Analysis of relative gene expression data using Real-time quantitative PCR and the 2[-DeltaDeltaC(T)] method. Methods 25: 402-408.

Moons A, Bauw G, Prinsen E, Van Montagu M, et al. (1995). Molecular and physiological responses to abscisic acid and salts in roots of salt-sensitive and salt-tolerant indica rice varieties. Plant Physiol.107: 177-186.

Parchmann S, Gundlach H and Mueller MJ (1997). Induction of 12-oxo-phytodienoic acid in wounded plants and elicited plant cell cultures. Plant Physiol.115: 1057-1064.

Shan DP, Huang JG, Yang YT, Guo YH, et al. (2007). Cotton GhDREB1 increases plant tolerance to low temperature and is negatively regulated by gibberellic acid. New Phytol. 176: 70-81.

Stenzel I, Hause B, Miersch O, Kurz T, et al. (2003). Jasmonate biosynthesis and the allene oxide cyclase family of Arabidopsis thaliana. Plant Mol. Biol. 51: 895-911.

Stumpe M and Feussner I (2006). Formation of oxylipins by CYP74 enzymes. Phytochem. Rev. 3: 347-357.

Szabados L and Savouré A (2010). Proline: a multifunctional amino acid. Trends Plant Sci. 15: 89-97.

Verbruggen N and Hermans C (2008). Proline accumulation in plants: a review. Amino Acids 35: 753-759.

Wang QY, Guan YC, Wu YR, Chen HL, et al. (2008). Overexpression of a rice OSDREB1F gene increases salt, drought, and low temperature tolerance in both Arabidopsis and rice. Plant Mol. Biol. 67: 589-602.

Wasternack C and Hause B (2002). Jasmonates and octadecanoids: signals in plant stress responses and development. Prog. Nucleic Acid Res. Mol. Biol. 72: 165-221.

Wu Q, Wu JJ, Sun HJ, Zhang D, et al. (2011). Sequence and expression divergence of the $A O C$ gene family in soybean: insights into functional diversity for stress responses. Biotechnol. Lett. 33: 1351-1359.

Yang A, Dai XY and Zhang WH (2012). A R2R3-type MYB gene, OsMYB2, is involved in salt, cold, and dehydration tolerance in rice. J. Exp. Bot. 63: 2541-2556.

Zheng XN, Zhen B, Lu GJ, and Han B (2009). Overexpression of a NAC transcription factor enhances rice drought and salt tolerance. Biochem. Biophys. Res. Commun. 379: 985-989.

Ziegler J, Stenzel I, Hause B, Maucher H, et al. (2000). Molecular cloning of allene oxide cyclase. The enzyme establishing the stereochemistry of octadecanoids and jasmonates. J. Biol.Chem. 275: 19132-19138. 